Educa-UMCH, 15, enero - junio 2020 https://doi.org/ 10.35756/educaumch.202015.129 ISSN: 2617-0337

\title{
Consecuencias psicológicas en mujeres víctimas de violencia de pareja
}

\section{Psychological consequences in women victims of partner violence}

Recibido: 20 de noviembre 2019, Evaluado: 27 de enero 2020, Aceptado: 30 de abril 2020

José Luis Colque Casas

psicjosecol@hotamil.com

https://orcid.org/0000-0002-6796-6868

Instituto de Investigación en psicología Forense y Criminología

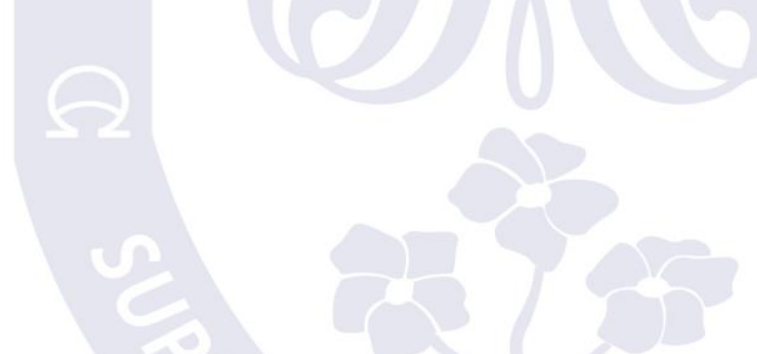

\section{Como citar el artículo}

Colque Casas, J. (2020). Consecuencias psicológicas en mujeres víctimas de violencia de pareja. Revista EDUCA UMCH, (15), 64-79 https://doi.org/10.35756/educaumch.202015.129

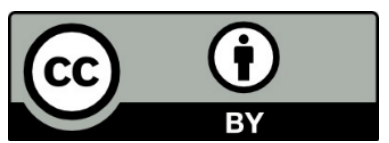

Esta obra está bajo una licencia internacional Creative Commons Atribución 4.0.

revistaeduca@umch.edu.pe

revistas.umch.edu.pe 


\section{Resumen}

El estudio tuvo como objetivo general determinar las consecuencias psicológicas de las mujeres víctimas de violencia por parte de su pareja. El diseño de estudio fue no experiental, transversal de alcance descriptivo. Se trabajo con una muestra de 108mujeres que denunciaron a sus parejas por violencia en la provincia constitucional del Callao durante los años 2018 y 2019, cuya muestra estuvo constituida por 108 mujeresvíctimas de violencia por parte de su pareja. Se utilizó la Lista de Chequeo de Síntomasde Distrés SCL-90 R de Derogatis, la cual identifica síntomas psicológicos mediante nueve dimensiones y tres índices globales de distrés. Entre los resultados en cuanto al sufrimiento psicológico en las víctimas de maltrato por parte de sus parejas: esta es superior a la de los pacientes psiquiátricos ambulatorios. Por otra parte, en cuanto a las dimensiones de síntomas primarios, sobresalen: la depresión $(X=1,81)$, la obsesión - compulsión $(X=1,74)$, la ansiedad $(X=1,70)$ y la somatización $(X=1,69)$. En tanto que los índices globales totales: las mujeres víctimas de maltrato presentan en promedio 56 síntomas positivos. Y se llegói a concluir que los síntomas psicológicos que presentan las mujeres víctimas de violencia por parte de su pareja se encuentran por encima de la población psiquiátrica ambulatoria $(52,03)$, la cual se respalda con la cantidad promedio de síntomas positivos encontrados (56). Las dimensiones que sobresalen en estas mujeres víctimas por parte de su pareja son: la depresión, obsesión - compulsión, ansiedad y somatización.

Palabras clave: consecuencias psicológicas, mujeres víctimas, violencia de pareja.

\section{Summary}

The study aimed to determine the psychological consequences of women victims of violence by their partner. The study design was non-experimental, cross-sectional in descriptive scope. We worked with a sample of 108 women who denounced their partnersfor violence in the constitutional province of Callao during the years 2018 and 2019, whose sample consisted of 108 women victims of violence by their partner. Derogatis SCL-90 R District Symptoms Checklist was used, which identifies psychological symptoms using nine dimensions and three global indexes of distress. Among the resultsregarding psychological suffering in victims of abuse by their partners: this is superior tothat of outpatient psychiatric patients. On the other hand, regarding the dimensions of primary symptoms, the following stand out: depression (X $=1.81)$, obsession - compulsion $(X=1.74)$, anxiety $(X=1.70)$ and somatization $(X=1.69)$. While the total global indexes: women victims of abuse have an average of 56 positive symptoms. And it was concluded that the psychological symptoms presented by women victims of violence by their partners are above the outpatient psychiatric population (52.03), which is supported by the average number of positive symptoms found (56). The dimensions that stand out in these women victims by their partner are: depression, obsession - compulsion, anxiety and somatization.

Keywords: psychological consequences, women victims, partner violence. 


\section{Introducción}

La violencia en contra de las mujeres es una dura realidad que nos viene tocando diariamente, Alencar-Rodriguez (2012). La violencia en contra de las mujeres es un fenómeno universal, heterogéneo, que abarca a todas las capas de la sociedad y que afectan en todas las edades.

El Perú no es ajeno a la violencia en contra de las mujeres, el último reporte del Instituto de estadística e informática (INEI, 2018), en relación a la violencia de pareja y familia, refieren que el $65,4 \%$ del total de las mujeres encuestadas fueron víctimas de algún tipo de violencia en algún momento de su vida, esto quiere decir que de cada 10 mujeres, 6 mujeres sufrieron algún tipo de violencia (psicológica, física, o sexual), siendola violencia psicológica la de mayor prevalencia $(61.5 \%)$, seguido de la violencia física $(30,6 \%)$ y la violencia sexual $(6,5 \%)$. Las estrategias psicológicas que utiliza el agresor en contra de las mujeres es el control $(57,1 \%)$, el acoso $(40,4 \%)$, los celos $(37,9 \%)$ y lashumillaciones $(19 \%)$.

Rajini, Kamesh Vell y Senthil (2014), realizaron un estudio descriptivo de corte transversal en 380 mujeres y encontraron que las características de las mujeres estudiadasfueron que el 52,6\% había sufrido violencia doméstica, siendo la edad de mayor prevalencia entre los 31 a 40 años y estando casadas el $43 \%$ (entre 6 a 10 años).

La Organización Mundial de la Salud (2005), define la violencia como: “el uso intencional de la fuerza o el poder físico, de hecho, o como amenaza contra uno mismo, otra persona o un grupo o comunidad, que cause o tenga muchas probabilidades de causarlesiones, muerte, daño psicológico, trastorno del desarrollo o privaciones".

En caso de la violencia psicológica, la normatividad peruana (Congreso de la República, 2015) la define como: "la acción o conducta, tendiente a controlar o aislar a la persona contra su voluntad, a humillarla, avergonzarla, insultarla, estigmatizarla o estereotiparla, sin importar el tiempo que se requiera para su recuperación".

Alberdi (2002), Loring (1994), Tolman (1989), O'Leary (1999), coinciden en señalar que en la violencia psicológica se usan una serie de estrategias (la intimidación, desvalorización, los insultos, la limitación de acceso a los recursos, los celos, etc.) que sedan de forma continua y sistemática, cuyo objetivo es tener el control total sobre la víctima por lo cual el agresor debe destruir la autoestima y la seguridad.

Marshall (1999), en relación a la violencia psicológica, refiere que esta se puede ejercer por conductas obvias que se reconocen y describen fácilmente (por ejemplo, agresión verbal, comportamientos de control), y conductas sutiles que tienen menos probabilidades de ser reconocidos como psicológicamente abusivos y estas se pueden disimular por patrones y modelos culturales y sociales que la invisibilizan, como el reparto de roles tradicionales en la pareja, costumbres o particularidades de los hombres.

Por su parte Calvete, Corral y Estévez (2005) describieron cuatro estrategias de maltrato psicológico: (1) aislamiento hostil (por ejemplo, ignorar y actuar de forma fría odistante respecto 
a la mujer), (2) conductas de intimidación (amenazar con emplear la violencia física o destruir intencionalmente las propiedades de la víctima), (3) denigración(insultos, utilizar el pasado de la víctima para avergonzarla, humillarla en público, acosomoral), y (4) conductas restrictivas (aislar a la mujer de su familia y amistades, impedirleel acceso al dinero, no dejarle trabajar o estudiar, etc.).

Echeburúa y Corral (2005), refiere que la violencia familiar y en especial contra las mujeres se configura como una situación de estrés crónico, enerando en la víctima, sentimientos de culpa y sufrimiento.

Biezma (2007), Buss (1961), Echeburúa y Corral (2005), Torres (2008), Checa (2010) y OrtizTallo (2013), coinciden en señalar que la violencia psicológica impacta negativamente en la salud física, emocional y social de la víctima, pero que esto va depender de la frecuencia y la severidad del maltrato.

Dutton (1996), refiere que no existe un perfil único de las mujeres que sufren violencia por parte de sus parejas y que las reacciones psicológicas que presentan, son respuestas psicológicas circunscritas a la dinámica de violencia, estas respuestas en algunos casos no van a cumplir los criterios de un diagnóstico "clínico", sin embargo lasrespuestas que presente la víctima son relevantes para el contexto legal, como por ejemplo la amnesia, la minimización, los sentimientos de culpa, etc, que puede presentar al momento de narrar la dinámica de violencia.

Por su parte Tolman (1989) y O'Leary (1999), refieren que la violencia contra las mujeres produce daño psicológico, las cuales se manifiestan con sentimientos de tristeza, ansiedad, inseguridad, invalidez, desamparo, baja autoestima, sentimientos de culpa, frustración y miedo.

Torres (2008), hace una síntesis muy interesante sobre los efectos del maltrato psicológico, por ejemplo: el aislamiento puede generar en la víctima dependencia y privación de apoyo social; el abuso verbal, la intimidación y las amenazas generan intimidación, miedo, ansiedad, terror y sumisión; el abuso emocional (desvalorización, tratar a la persona como una persona inferior, etc), puede generar inseguridad y baja autoestima; la negación y la culpabilización pueden generar en la víctima inseguridad e indefensión; y el acoso genera intimidación, miedo y ansiedad.

Echeburúa, Corral y Amor (2004), en relación con el daño psicológico, esta se refiere, por un lado, a las lesiones psíquicas agudas y que pueden remitir con el paso del tiempo, el apoyo social o un tratamiento psicológico adecuado; y, por otro, a las secuelas emocionales que persisten en la persona de forma crónica, como consecuencia del suceso sufrido y que interfiere negativamente en su vida cotidiana.

En la investigación de Mattos, Montalvo y Oviedo (2016), sobre daño psicológico en víctimas de violencia conyugal, encontraron que los valores de las dimensiones primarias del SCL-90$\mathrm{R}$ se encuentran por encima del cohorte evidenciando indicadores clínicos elevados de depresión (70,97\%), ansiedad fóbica (70,97\%), y somatización $(67,74 \%)$. 
En el estudio desarrollado por Avdibegović y Sinanović (2006) con víctimas de violencia doméstica en Bosnia y Herzegovina, donde se utilizó el SCL-90-R, encontraron que las dimensiones primarias en estas mujeres abusadas tuvieron una tasa significativamente más alta en depresión, somatización, sensibilidad interpersonal, obsesión - compulsión, ansiedad y tendencia paranoide en comparación con las mujeres que no fueron abusadas.

Jaramillo, Uribe, Ospina, y Cabarcas (2003), desarrollaron en Medellín un estudiocon mujeres que sufrieron violencia doméstica, donde se les aplicó el SCL-90-R, encontrando que los promedios de las dimensiones de los síntomas primarios se encuentran por encima de los valores referenciales establecidos por Derogatis para mujeres no pacientes y cercanos a los de las mujeres pacientes psiquiátricas en tratamiento ambulatorio. Dentro de las dimensiones de los síntomas primarios quesobresalen son: depresión $(X=1,70 ; \mathrm{t}=20.73)$, ideación paranoide $(\mathrm{X}=1,48 ; \mathrm{t}=18.45)$, obsesión - compulsión $(\mathrm{X}=1.41 ; \mathrm{t}=18.11)$, sensibilidad interpersonal $(\mathrm{X}=1.35 ; \mathrm{t}=19.91)$ ysomatización $(\mathrm{X}=1.31 ; \mathrm{t}=14.96)$.

\section{Diseño}

\section{Metodología}

Se siguió un diseño descriptivo transversal, cuyo objetivo es describir a la población de estudio e identificar los síntomas que presentó durante los últimos siete días al momento de la evaluación. El muestreo utilizado fue no probabilístico, donde los sujetos fueron seleccionados por conveniencia dada la accesibilidad y proximidad con los sujetosinvestigados.

\section{Población y muestra}

La población estuvo constituida por las mujeres de la provincia constitucional del Callaoque denunciaron violencia por parte de sus parejas. La muestra estuvo constituida por 108mujeres que pasaron por evaluación psicológica en un servicio forense del Callao por orden judicial, fiscal y/o policial debido a una denuncia interpuesta por estas mujeres en contra de sus parejas o ex parejas. La edad de las evaluadas estuvo comprendida desde los 14 hasta los 66 años.

\section{Instrumento}

Para el presente estudio se utilizó la lista de síntomas SCL-90-R de Derogatis, la cual identifica el estado de los síntomas psicológicos. Las personas señalan en una escala tipo Likert, la intensidad del malestar que les causa cada uno de los síntomas de acuerdo con un puntaje que oscila entre 0 (ausencia total de molestia relacionada con el síntoma) hasta 4 (molestia máxima).

La presencia o ausencia de los síntomas en el cuestionario se restringe a la experiencia reciente,

revistaeduca@umch.edu.pe

revistas.umch.edu.pe 
es decir, se espera que el sujeto haga una valoración de las molestias ocasionadas por cada síntoma durante los días inmediatos previos a la entrevista, incluyendo el día de la evaluación, aunque Derogatis afirma que no encontró diferencias significativas al aumentar el rango temporal hasta los quince días anteriores de la evaluación. Las dimensiones de síntomas primarios son: somatización, obsesivo- compulsivo, sensibilidad interpersonal, depresión, ansiedad, hostilidad, ansiedad fóbica, ideas paranoides y psicoticismo.

Los índices globales de distrés proveen datos para la valoración total del estadopsicológico de la persona que estamos evaluando y nos proporciona un resumen tanto dela sintomatología como del malestar psicológico, y reflejan distintos aspectos del desorden psicológico. El índice global de severidad (GSI) refleja la intensidad delsufrimiento psicológico y psicosomático de la evaluada. El índice total de síntomas positivos (PST) refleja el número de síntomas positivos, y refleja la amplitud y diversidadde la sintomatología presente y finalmente, el índice de síntomas positivos (PSDI) indicala intensidad del síntoma o la característica de la evaluada para experimentar el sufrimiento psicológico, esto es, informa si la evaluada maximiza o minimiza susrespuestas.

Para este estudio se midió la consistencia interna de la SCL-90-R mediante el alpha de Cronbach, que fue de 0,98 .

\section{Resultados}

\section{Características sociodemográficas}

Las edades de las mujeres que denuncian maltrato de parte de su pareja fluctuaronentre 14 y 66 años, siendo las edades más frecuentes, la comprendida entre los 30 a 36 años con un 26,9\%, seguida por las de 37 a 44 años con un 19,4\% (Ver Tabla 1).

\section{Tabla 1}

Edad

\begin{tabular}{lll}
\hline Edad & Frecuencias $=\mathrm{N}$ & Porcentaje Válido $=\%$ \\
\hline $14-21$ & 13 & 12,0 \\
$22-29$ & 21 & 19,4 \\
$30-36$ & 29 & 26,9 \\
$37-44$ & 21 & 19,4 \\
$45-51$ & 15 & 13,9 \\
52 a más & 9 & 8,3 \\
\hline
\end{tabular}

En la tabla 2, en cuanto a su estado civil, el 36,8\% de las mujeres que denuncian ex violencia por parte de su pareja al momento de la denuncia se encontraban en calidad de conviviente $(36,8 \%)$, seguido de las casadas $(29,2 \%)$ y convivientes $(22,6 \%)$. 
Tabla 2

Estado civil

\begin{tabular}{lll}
\hline Estado civil & Frecuencias $=\mathrm{N}$ & Porcentaje Válido $=\%$ \\
\hline Soltera & 9 & 8,5 \\
Casada & 31 & 29,2 \\
Conviviente & 24 & 22,6 \\
Divorciada & 3 & 2,8 \\
Ex conviviente & 39 & 36,8 \\
\hline Total & 106 & 100 \\
\hline
\end{tabular}

En la tabla 3, en cuanto a su grado de instrucción, estas mujeres en su gran mayoría, el $66,4 \%$ refieren haber culminado sus estudios secundarios.

Tabla 3

Grado de Instrucción

\begin{tabular}{lll}
\hline Grado de Instrucción & Frecuencias =N & Porcentaje válido =\% \\
\hline Primaria completa & 1 & 0,9 \\
Secundaria incompleta & 10 & 9,3 \\
Secundaria completa & 71 & 66,4 \\
Superior incompleto & 13 & 12,1 \\
Superior completo & 12 & 11,2 \\
\hline Total & 107 & 100.00 \\
\hline
\end{tabular}

En la tabla 4, en cuanto al tiempo de relación, la mayoría de las mujeres que denunciaron tienen una relación de pareja mayor a 10 años $(37,3 \%)$, y las que menos denuncian son aquellas personas que tienen una relación de 1 año a 2 años $(6,9 \%)$.

Tabla 4

Tiempo de relación

\begin{tabular}{|l|c|c|}
\hline Tiempo de relación & Frecuencias $=\mathrm{N}$ & Porcentaje válido $=\%$ \\
\hline Menos de un año & 20 & 19,6 \\
\hline De un año un mes a dos años & 7 & 6,9 \\
\hline De dos años un mes a cinco años & 14 & 13,7 \\
\hline De cinco años un mes a diez años & 23 & 22,5 \\
\hline De diez años un mes a más & 38 & 37,3 \\
\hline
\end{tabular}

En la tabla 5, en cuanto al tipo de abuso sufrido, la mayoría de las personas encuestadas refiere que la estrategia que usaba su agresor era de dominio - control $(62,9 \%)$ y mixto $(25,8 \%)$

revistaeduca@umch.edu.pe

revistas.umch.edu.pe 
Tabla 5

Tipo de abuso psicologico

\begin{tabular}{lll}
\hline Tipo de abuso psicológico & Frecuencias =N & Porcentaje válido $=\%$ \\
\hline Abuso emocional verbal & 11 & 11,3 \\
Abuso de tipo control - dominio & 61 & 62,9 \\
Abuso mixto & 25 & 25,8 \\
\hline
\end{tabular}

\section{Resultado de las escalas clínicas e índices globales del SCL-90-R}

En la tabla 6, se midió la consistencia interna de la SCL-90-R mediante el alpha de Cronbach, que fue de 0,98. Además, se calcularon los promedios de las nueve dimensiones de síntomas primarios y tres índices globales de distrés, los cuales se comparan con valores de referencia establecidos por Derogatis y Jaramillo et al. (2006) que midió el distrés psicológico en mujeres maltratadas, en Medellín. Las mediasobtenidas en la muestra son significativamente mayores que las de las mujeres no pacientes, para todas las dimensiones de los síntomas se obtuvo un $\mathrm{p}<0.0001$. Igualmente, cuando los resultados se comparan con los valores de las mujeres maltratadas en Medellín, se observan que los valores de las muestras son muy cercanos a la muestra peruana.

Tabla 6

Promedio de las dimensiones de síntomas primario e índices globales en mujeres de referencia de Derogatis, mujeres de Medellín y la muestra de mujeres peruana Valores de referencia

\begin{tabular}{|c|c|c|c|c|}
\hline $\begin{array}{lc} & \mathrm{Mu} \\
& \mathrm{pac} \\
\text { Dimensiones de síntomas } & \mathrm{adu} \\
\end{array}$ & $\begin{array}{l}\text { Mujeres no } \\
\text { acientes } \\
\text { dultas }\end{array}$ & $\begin{array}{l}\text { Mujeres } \\
\text { pacientes } \\
\text { psiquiátricas } \\
\text { adultas }\end{array}$ & $\begin{array}{l}\text { Muestra de } \\
\text { mujeres de } \\
\text { Medellín } \\
\text { Colombia }\end{array}$ & $\begin{array}{l}\text { Muestra de } \\
\text { Mujeres } \\
\text { Perú }\end{array}$ \\
\hline Somatización (SOM) & 0,43 & 0,99 & 1,31 & 1,69 \\
\hline $\begin{array}{l}\text { Obsesivo Compulsivo (OBS- } \\
\text { COM) }\end{array}$ & 0,44 & 1,51 & 1,41 & 1,74 \\
\hline $\begin{array}{l}\text { Sensibilidad interpersonal } \\
\text { (INT) }\end{array}$ & 0,35 & 1,44 & 1,35 & 1,39 \\
\hline Depresión (DEP) & 0,46 & 1,94 & 1,70 & 1,81 \\
\hline Ansiedad (ANS) & 0,37 & 1,59 & 1,11 & 1,70 \\
\hline Hostilidad (HOS) & 0,33 & 1,17 & 1,25 & 0,82 \\
\hline Ansiedad Fóbica (FOB) & 0,19 & 0,81 & 0,79 & 1,34 \\
\hline Ideación Paranoide (PAR) & 0,34 & 1,21 & 1,48 & 1,64 \\
\hline Psicoticismo (PSIC) & 0,15 & 0,98 & 0,95 & 1,04 \\
\hline Índice Global de Severidad (IGS) & 5) 0,36 & 1,31 & 1,29 & 1,52 \\
\hline Total, de síntomas positivos (PST & T) 22 & 52,03 & 47,33 & 56 \\
\hline $\begin{array}{l}\text { Índice de alteración de síntomas } \\
\text { positivos } \\
\text { (PSDI) }\end{array}$ & 1,37 & 2,22 & 2,38 & 2,31 \\
\hline
\end{tabular}


En la tabla 7, en cuanto a las dimensiones de síntomas primarios se puede observar que la escala "Depresión" fue la que presentó la mayor frecuencia de síntomas $\left(X=1,81 ; S_{X}=0.95\right)$, seguido de "Obsesión - compulsión" $(\mathrm{X}=1,74 ; \mathrm{S} x=0.92)$ y posteriormente de "Somatización" $\left(X=1,69 ; S_{X}=0.99\right)$. La sintomatología de menor frecuencia fue la de "Hostilidad" $(X=0,82$; $\left.\mathrm{S}_{\mathrm{X}}=0.72\right)$.

Tabla 7

Promedio de las dimensiones de síntomas primarios

\begin{tabular}{lcll}
\hline Indices & $\mathrm{N}$ & Media & Desv. Est. \\
\hline Somatización & 108 & 1,69 & 0,99 \\
Obsesión - compulsión & 108 & 1,74 & 0,92 \\
Sensibilidad interpersonal & 108 & 1,39 & 0,92 \\
Depresión & 108 & 1,81 & 0,95 \\
Ansiedad & 108 & 1,70 & 1,03 \\
Hostilidad & 108 & 0,82 & 0,72 \\
Ansiedad fóbica & 108 & 1,34 & 1,04 \\
Ideación paranoide & 108 & 1,64 & 0,99 \\
Psicoticismo & 108 & 1,04 & 0,87 \\
\hline
\end{tabular}

En la tabla 8, referido a los Índices Globales, del total de 90 síntomas de la SCL-90-R, las mujeres denunciantes informaron presentar en promedio 56 síntomas positivos (PST); en cuanto al índice de severidad (GSI) el promedio se encuentra en 1,52; sobre la intensidad de los síntomas (PSDI) de 0-4, las mujeres arrojaron un promedio de 2,31. Estos resultados ubican a las mujeres en un perfil de distrés muy cercano al de pacientespsiquiátricos ambulatorios.

Tabla 8

Índices Globales

\begin{tabular}{lccc}
\hline Dimensiones de síntomas & N & Media & Desv. Est. \\
\hline Índice Global de Severidad (GSI) & 108 & 1,52 & 0,83 \\
Total, de síntomas positivos (PST) & 108 & 56 & 21,67 \\
Índice de intensidad de síntomas positivos108 & 2,31 & 0,57 \\
(PSDI) & & \\
\hline
\end{tabular}

En la tabla 9, referida a la Depresión, se observa que los ítems con mayor prevalencia son el ítem 31 "preocuparse demasiado por todo" $(\mathrm{X}=2,92 ; \mathrm{Sx}=1.197)$, ítem 30 "sentirse triste" $(X=2,82 ; \mathrm{Sx}=1.388)$, el ítem 20 "Llorar fácilmente $(X=2,21 ; \mathrm{Sx}=1.472)$ y el ítem 14 "sentirse baja de energía o decaída" $(X=2,18 ; \mathrm{Sx}=1.396)$. El ítem que obtuvo menor puntuación fue el ítem 15 "Pensamientos suicidas, o ideas de acabar con su vida" $\left(\mathrm{X}=0,64 ; \mathrm{S}_{\mathrm{X}}=1.197\right)$. 
Tabla 9

Depresión

\begin{tabular}{llll}
\hline Items & $\mathrm{N}$ & Media & Desv. Est. \\
\hline Ítem 5. Pérdida de deseo o de placer sexual & 101 & 1,86 & 1.609 \\
Ítem 14. Sentirse bajo de energías o decaído & 101 & 2,15 & 1.393 \\
Ítem 15. Pensamientos suicidas, o ideas de acabar con & 101 & 0,64 & 1.192 \\
su vida & 101 & 2,17 & 1.495 \\
Ítem 20. Llorar fácilmente & 101 & 1,71 & 1.571 \\
Ítem 22. La sensación de estar atrapado o como & & & \\
encerrado & 101 & 1,94 & 1.465 \\
Ítem 26. Culparse a sí mismo de todo lo que pasa & 101 & 2,08 & 1.572 \\
Ítem 29. Sentirse solo & 101 & 2,77 & 1.364 \\
Ítem 30. Sentirse triste & 101 & 2,92 & 1.197 \\
Ítem 31. Preocuparse demasiado por todo & 101 & 1,25 & 1.269 \\
Ítem 32. No sentir interés por nada & 101 & 1,35 & 1,474 \\
Ítem 54. Sentir desesperanzado con respecto al futuro & 101 & 1,70 & 1,449 \\
Ítem 71. Sentir que todo requiere un gran esfuerzo & 101 \\
Ítem 79. La sensación de ser inútil o no valer nada & 101 & 0,92 & 1,368 \\
\hline
\end{tabular}

En la tabla 10, referida a la Obsesión - Compulsión, se observa que los ítems con mayor prevalencia son los ítem 3, 10 y 46; donde sobresalen "Pensamientos, palabras o ideas no deseadasque no se van de su mente" $\left(X=2,04 ; S_{X}=1.435\right)$, "Preocupación acerca del desaseo, el descuido ola desorganización" $(\mathrm{X}=2,04 ; \mathrm{S}=1.407)$, y "Dificultad en tomar decisiones" ( $\left.\mathrm{X}=2,04 ; \mathrm{S}_{X}=1.303\right)$ respectivamente. El ítem que obtuvo menor puntuación fue el ítem 65 "Impulsos a tener que hacerlas cosas de manera repetida" (X=1,22; $\left.\mathrm{S}_{\mathrm{X}}=1.376\right)$.

Tabla 10

Obsesión - Compulsión

\begin{tabular}{lccc}
\hline Items & $\mathrm{N}$ & Media & Desv.Est. \\
\hline $\begin{array}{l}\text { İtem 3. Pensamientos, palabras o ideas no deseadas que no se } \\
\text { van desu mente }\end{array}$ & 101 & 2.04 & 1.435 \\
$\begin{array}{l}\text { Ítem 9. La dificultad para recorder las cosas } \\
\text { Ítem 10. Preocupación acerca del desaseo, el }\end{array}$ & 101 & 1.67 & 1.335 \\
descuido o ladesorganización & 101 & 2.04 & 1.407 \\
Ítem 28. Sentirse incapaz de hacer las cosas o terminar las tareas 101 & 1.63 & 1.214 \\
Ítem 38. Tener que hacer las cosas muy despacio para estar & 101 & 1.87 & 1.383 \\
seguro de & & & \\
que las hace bien & & & \\
Ítem 45. Tener que comprobar una y otra vez todo lo que hace & 101 & 1.49 & 1.262 \\
Ítem 46. Dificultad en tomar decisiones & 101 & 2.04 & 1.303 \\
Ítem 51. Que le quede la mente en blanco & 101 & 1.46 & 1.432 \\
Ítem 55. Tener dificultades para concentrarse & 101 & 1.88 & 1.366 \\
Ítem 65. Impulsos a tener que hacer las cosas de manera repetida 101 & 1.22 & 1.376 \\
\hline
\end{tabular}


En relación con la tabla 11, referida a la Ansiedad, se observa que los ítems con mayor prevalencia son el ítem 2 "Nerviosismo o agitación interior" $\left(X=2,50 ; S_{X}=1.172\right)$, ítem 33 "Sentirse temeroso" $\left(\mathrm{X}=2,36 ; \mathrm{S}_{\mathrm{X}}=1.346\right)$, e ítem 57 "Sentirse tenso o con los nervios de punta" (X=2,08; $\left.S_{X}=1.383\right)$. El ítem que obtuvo menor puntuación fue el ítem 86 "Pensamientos o imágenes estremecedoras o que le dan miedo" $\left(X=1,09 ; S_{X}=1.289\right)$.

Tabla 11

Ansiedad

\begin{tabular}{l|r|rl}
\hline Items & $\mathrm{N}$ & Media & $\begin{array}{l}\text { Desv. } \\
\text { Est. }\end{array}$ \\
\hline Ítem 2. Nerviosismo o agitación interior & 101 & 2.50 & 1.172 \\
Ítem 17. Temblores & 101 & 1.24 & 1.372 \\
Ítem 23. Tener miedo de repente y sin razón & 101 & 1.61 & 1.421 \\
Ítem 33. Sentirse temeroso & 101 & 2.36 & 1.346 \\
Ítem 39. Que su corazón palpite o vaya muy de prisa & 101 & 1.80 & 1.483 \\
Ítem 57. Sentirse tenso o con los nervios de punta & 101 & 2.08 & 1.383 \\
Ítem 72. Ataques de terror o pánico & 101 & 1.38 & 1.406 \\
Ítem 78. Sentirse tan inquieto que no puede si estar sentado & 101 & 1.13 & 1.309 \\
tranquilo & & & \\
Ítem 80. Presentimientos de que va a pasar algo malo & 101 & 1.92 & 1.528 \\
Ítem 86. Pensamientos o imágenes estremecedoras o que le & 101 & 1.09 & 1.289 \\
dan miedo & & & \\
\hline
\end{tabular}

En relación con la tabla 12, referida a los ítems adicionales, se observa que los ítems con mayor prevalencia son: el ítem 44 "problemas para dormir" $\left(X=2,12 ; S_{X}=1.575\right)$, ítem 66 "Sueño intranquilo" $\left(X=2,01 ; S_{X}=1.507\right)$, y el ítem 19 "poco apetito" $\left(X=1,81 ; S_{X}=1.370\right)$.

Tabla 12

Items adicionales

\begin{tabular}{lrrl}
\hline Items & $\mathrm{N}$ & Media & $\begin{array}{l}\text { Desv. } \\
\text { Est. }\end{array}$ \\
\hline Ítem 19. Poco apetito & 101 & 1.81 & 1.370 \\
Ítem 44. Problemas para dormir & 101 & 2.12 & 1.575 \\
Ítem 59. Pensamientos acerca de la muerte o el & 101 & 0.95 & 1.314 \\
morirse & & & \\
Ítem 60. Comer en exceso & 101 & 0.99 & 1.196 \\
Ítem 64. Despertarse muy temprano & 101 & 1.69 & 1.495 \\
Ítem 66. Sueño intranquilo & 101 & 2.01 & 1.507 \\
Ítem 89. Sentimientos de culpa & 101 & 1.64 & 1.519 \\
\hline
\end{tabular}

En relación a la tabla 13, referido a los ítems de riesgo psicopatológico, se observa que los ítemscon mayor prevalencia son: el ítem 35 "Tener la impresión de que los demás se dan 
cuenta de sus pensamientos" $(\mathrm{X}=1,29 ; \mathrm{Sx}=1.374)$. El ítem que obtuvo menor puntuación fue el ítem 16 "oír voces que otras personas no oyen" $(\mathrm{X}=1,09 ; \mathrm{Sx}=1.289)$.

Tabla 13

Items de riesgo psicopatológico

\begin{tabular}{|c|c|c|c|}
\hline 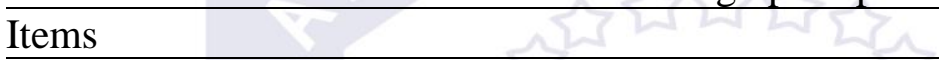 & $\mathrm{N}$ & Media & Desv. Est. \\
\hline $\begin{array}{l}\text { Ítem 15. Pensamientos suicidas, o ideas de acabar con } \\
\text { su vida }\end{array}$ & 101 & 0.64 & 1.192 \\
\hline Ítem 16 . Oír voces que otras personas no oyen & 101 & 0.36 & 0.861 \\
\hline $\begin{array}{l}\text { Ítem } 35 \text {. Tener la impresión de que los demás se dan } \\
\text { cuenta de suspensamientos }\end{array}$ & 101 & 1.29 & 1.374 \\
\hline $\begin{array}{l}\text { Ítem } 84 \text {. Tener pensamientos sobre el sexo que le } \\
\text { inquietan bastante }\end{array}$ & 101 & 0.85 & 1.274 \\
\hline $\begin{array}{l}\text { Ítem } 85 \text {. La idea de que debería ser castigada por sus } \\
\text { pecados }\end{array}$ & 101 & 0.71 & 1.068 \\
\hline
\end{tabular}

\section{Discusión}

El presente estudio tuvo como objetivo fundamental determinar los efectos del maltrato sobre la salud psicológica de las mujeres víctimas de violencia por parte de su pareja.

La franja de edad con mayor prevalencia se encuentra entre los 30 - 36 años, siendo las casadas y las que mantienen una relación de convivencia, las que denuncian con mayor frecuencia, dichos resultados coinciden con lo que establecen otros autores (Rajini, Kamesh Vell, y Senthil (2014).

En cuanto a las estrategias de violencia psicológica que usan los agresores en contra de las mujeres, coincide con los estudios desarrollados por diversos autores, resaltando las estrategias de control - dominio (Dutton, 1996, Alberdi, 2002, Tolman, 1989, y O’Leary, 1999).

En cuanto a las consecuencias psicológicas producto del maltrato, diversos autorese investigaciones coinciden en señalar que los síntomas que presentan las víctimas de violencia de pareja, suelen ser dispersos y que estos deben de estar delimitadas a la dinámica de violencia (Dutton, 1996).

En el presente estudio las dimensiones de síntomas primarios que resaltan en las víctimas de violencia por parte de su pareja son: la depresión, la ansiedad, obsesión compulsión y la somatización, los cuales coinciden con los estudios desarrollados por Mattos, Montalvo, y Oviedo (2016); Avdibegović y Sinanović (2006), y Jaramillo, Uribe, Ospina y Cabarcas (2003).

Dentro de los síntomas depresivos que destacan, se encuentra la preocupación, la revistaeduca@umch.edu.pe revistas.umch.edu.pe 
tristeza, el llanto, el decaimiento, la pérdida de energía, y los sentimientos de soledad y de culpa. En cuanto a los síntomas ansiosos resaltan: el nerviosismo, el temor y miedo,la tensión y los pensamientos negativos de que algo malo puede suceder. En cuanto a los síntomas obsesivos - compulsivo, los que predominan son los pensamientos o ideas nodeseados que no se van de la mente, las dificultades para poder tomar una descisión, preocupación de que todo esté ordenado y dificultades para poder concentrarse. En cuantoa los síntomas de somatización, los síntomas que priman son sentir un nudo en la garganta, los dolores de cabeza, debilidad en alguna de las partes del cuerpo y dolores musculares.

\section{Conclusiones}

Se halló que la mayoría de las mujeres que denuncia violencia o maltrato por partede sus parejas o ex parejas se encuentran entre los 30 a 36 años. En cuanto a su grado deinstrucción, más de la mitad de la muestra estudiada cuenta con secundaria completa $(66,4 \%)$.

Con respecto al estado civil, se encontró que quienes denuncian con mayor prevalencia son las casadas y convivientes $(51,8 \%)$ y ex convivientes $(36,8 \%)$.

En tanto al uso del SCL-90-R como un instrumento de tamizaje en mujeres que sufren violencia, demuestra ser un buen predictor, ya que su nivel de confiabilidad es muyalta $(0,98)$.

El presente estudio corrobora lo planteado por Dutton (1996, 2000), ya que no existe un perfil único de síntomas en las mujeres que sufren violencia por parte de sus parejas, siendo esta muy variable.

Sobre las consecuencias psicológicas de las mujeres víctimas que denuncian maltrato por parte de su pareja, estas son muy superiores a las mujeres que no sufren violencia, sobresaliendo las dimensiones de depresión, obsesión - compulsión, ansiedady somatización.

Finalmente, el presente estudio plantea dos reflexiones finales: La primera es queel SCL-90-R es un buen instrumento de tamizaje para detección de alteraciones psicológicas en víctimas de violencia de pareja, sin embargo, queda mucho por seguir investigando. Y la segunda, se ha confirmado que las consecuencias psicológicas que padecen las víctimas de violencia por parte de sus parejas se encuentran por encima de las personas que no viven violencia.

\section{Referencias}

Alberdi, I. (2002). La violencia doméstica. Informe sobre los malos tratos a mujeres en España. En Pérez del Campo, A. Las jóvenes frente a la violencia de género. Revista de estudios de juventud, 86(5). Pp: 83-98. Recuperado de: http://pmayobre.webs.uvigo.es/pdf/la_violencia_domestica_informe_malos_trat os_a_mujeres_en_espana.pdf

Aldana, J. (2012). Análisis interdisciplinario de las diversas formas de violencia contra la mujer. revistaeduca@umch.edu.pe revistas.umch.edu.pe 
Guatemala: Cara Parens.

Alencar-Rodriguez, R. y Cantera, L. (2012). Violencia de Género en la pareja: una revisión Teórica. Recuperado de: file:///C:/Users/lenovo/Downloads/DialnetViolenciaDeGeneroEmCasais-5163211.pdf

Aragonès de la Cruz, R., Farran i Porté, M., Guillén, J., y Rodríguez, L (2018). Perfil psicológico de víctimas de violencia de género, credibilidad y sentencias. Recuperado de:

http://cejfe.gencat.cat/web/.content/home/recerca/cataleg/crono/2018/victimesViolenci a_ES.pdf

Arenas, L. (2012). Violencia psicológica y mantenimiento en la relación de pareja. [Tesispara optar el titulo de licenciada en Psicología]. Recuperado de: http://tesis.pucp.edu.pe/repositorio/bitstream/handle/123456789/1692/ARENASROM EROLINAVIOLENCIA.pdf?sequence

Avdibegović, E. y Sinanović, O. (2006). Consequences of Domestic Violence onWomen's Mental Health in Bosnia and Herzegovina. Croat Med J. Oct; 47(5).Pp:730-741. Recuperado de: https://www.ncbi.nlm.nih.gov/pubmed/17042065

Biezma, J. (2007). Violencia hacia la pareja: revisión teórica. Universidad Complutensede Madrid. Psicopatología Clínica, Legal y Forense. Volumen 7. Pp:77-95.

Biezma, R. (2007). Psicopatología clínica, legal. Recuperado de: https://webs.ucm.es/BUCM/compludoc//S/10904/15769941_1.htm

Buss, A. (1961). The Psychology of Aggression. New York: First Edition

Calvete, E., Corral, S y Estévez, A (2005), Desarrollo de un inventario para evaluar el abuso psicológico en las relaciones de pareja. Clínica y Salud, 2005. 16(3). Pp: 203-221. ISSN: 1135-0806, recuperado de:https://www.redalyc.org/pdf/1806/180617759001.pdf

Canabal, G., Gonzales, M., Humphreys, J., De León, N., y Gonzáles, S. (2008). Violenciade pareja y salud de las mujeres que consultan a las comisarías de familia, Cali, Colombia. Recuperado de: http://www.scielo.org.co/pdf/iee/v27n2/v27n2a06.pdf

Checa, M. (2010). Manual práctico de Psiquiatria Forense. España: Elsevier Masson.

Congreso de la República. (2015). Ley 30364: Ley para prevenir, sancionar y erradicar la violencia contra las mujeres y los integrantes del grupo familiar. Perú.

Derogatis, L. (2002). Manual SCL-90-R, Cuestionario de 90 síntomas. Madrid: TEA Ediciones. 
Dutton, D. y Kropp, P. (2000). “A Review of Domestic Violence Risk Instruments". En "Trauma, Violence and Abuse".1(2). Pp: 171-181.

Dutton, M. (1996). “La mujer maltratada y sus estrategias de respuesta a la violencia", en Edleson, J. y Eisikovits, Z. (comps.). Violencia doméstica: la mujer golpeada y la familia. Buenos Aires: Granica. 1997. Pp:153-178.

Echeburúa, E. Corral, P. y Amor, P. (2004). Evaluación del daño psicológico en las víctimas de delitos violentos. Psicopatologia Clínica, Legal y Forense. Vol. 4, 2004, pp. 227 244. Recuperado de: https://masterforense.com/pdf/2004/2004art19.pdf

Echeburúa, E. y Corral, P. (2005). ¿Cómo evaluar las lesiones psíquicas y las secuelas emocionales en las víctimas de delitos violentos? Recuperado de: http://masterforense.com/pdf/2005/2005art3.pdf

Instituto Nacional de Estadistica e Informatica - INEI. (2018). Perú: Indicadores de violencia familiar y sexual, 2000 - 2017. Recuperado de: https://www.inei.gob.pe/media/MenuRecursivo/publicaciones_digitales/Est/Lib1 $\underline{465 / \text { libro.pdf }}$

Jaramillo, D., Uribe, T., Ospina, D., y Cabarcas, G. (2003). Medición de distrés psicológico en mujeres maltratadas, Medellín. Recuperado de: http://www.scielo.org.co/pdf/cm/v37n2/v37n2a07.pdf

Marshall, L. (1999). Effects of men's subtle and overt psychological abuse on low-income women. Violence and Victims, 14(1),69-88. Recuperado de: https://www.ncbi.nlm.nih.gov/pubmed/10397627

Mattos, Y., Montalvo, M., y Oviedo, A. (2016). Daño psicológico en mujeres víctimas de violencia conyugal. [tesis para optención de grado de magister]. Recuperado de: http://www.bibliotecadigital.usb.edu.co/bitstream/10819/3993/1/Da\%C3\%B1os\%20ps icol\%C3\%B3gicos\%20mujeres_Yuris\%20Mattos\%20Q 2016.pdf

Nuñez, J. y Carbajal, H. (2004). Violencia Intrafamiliar. Abordaje integral a víctimas. Sucre. Tupac Katari.

O'Leary, K. (1999). Psychological abuse: a variable deserving critical attention in domestic violence. Violence and Victims, 14(1), 3-23. Recuperado de: https://www.ncbi.nlm.nih.gov/pubmed/10397623

Organización Mundial de la Salud - OMS (2019). Informe mundial sobre la violencia y la salud. Recuperado de:

https://www.who.int/violence_injury_prevention/violence/world_report/es/summary_e $\underline{\text { s.pdf }}$

revistaeduca@umch.edu.pe

revistas.umch.edu.pe 
Pynoos, R., Sorenson, S. y Steinberg, A. (1993). Interpersonal violence and traumatic stress reactions. En L. Goldberger y S. Breznitz (Eds). Handbook of stress: Theoretical and clinical aspects (2nd de.). New York. Free Press.

Rajini, S, Kamesh Vell, C, y Senthil, S (2014). Prevalence of domestic violence and health seeking behavior among women in rural community of puducherry - across sectional study. Int J Cur Res Rev | Vol 6 - Issue 16. Recuperado de: https://www.semanticscholar.org/paper/PREVALENCEOFDOMESTICVIOLENCEAND-HEALTH-SEEKINGRajiniVell/21da3f5b632dcaeb8c06e8c1dfd582a9a074e689

Tolman, R. (1989). The development of measure of Psychological maltreatment of woman by their male parners. Violence and victims, 4 (3), pág. 159 - 177. Springer Publishing Company. Recuperado de: https://www.ncbi.nlm.nih.gov/pubmed/2487132

Torres, A. (2014). Violencia de pareja: detección, personalidad y bloqueo de la huida [Tesis doctoral de la universidad de Barcelona]. Recuperado de: https://www.tesisenred.net/handle/10803/313041 